\title{
Marcel Bernos, Femmes et gens d'Église dans la France classique, XVIIe-XVIIIe siècles
}

\author{
Robert Sauzet
}

\section{OpenEdition}

\section{Journals}

Édition électronique

URL : http://journals.openedition.org/abpo/1447

DOI : $10.4000 / a b p o .1447$

ISBN : 978-2-7535-1490-4

ISSN : 2108-6443

Éditeur

Presses universitaires de Rennes

Édition imprimée

Date de publication : 20 juillet 2003

Pagination : 248-250

ISBN : 978-2-86847-874-0

ISSN : 0399-0826

\section{Référence électronique}

Robert Sauzet, « Marcel Bernos, Femmes et gens d'Église dans la France classique, XVIle-

XVIIle siècles », Annales de Bretagne et des Pays de l'Ouest [En ligne], 110-2 | 2003, mis en ligne le 20 juillet 2005, consulté le 01 mai 2019. URL : http://journals.openedition.org/abpo/1447 ; DOI : 10.4000/ abpo. 1447 
au moment où l'autorité royale se fait plus présente, notamment pour exiger des fournitures de salpêtre puis, de plus en plus, des contributions financières. Dans les dernières pages de l'ouvrage, enfin, Daniel Leloup étudie " Nantes, le château et la trace profonde de la ville close " (p. 359-368). Il rapproche avec justesse l'implantation de ce château au bord du fleuve avec celle du Louvre à Paris. La ville et le château ne changent guère jusqu'au milieu du $\mathrm{XVIII}^{\mathrm{e}}$ siècle où une active politique d'urbanisme amène progressivement le démantèlement des fortifications et le rattachement du château à la terre ferme et à la ville. Peu à peu, la construction a été dénaturée dans ses fonctions comme dans son image mais son environnement urbain a subsisté puisque les grandes réalisations urbanistiques ont été réalisées, à l'exception du percement de la rue de Strasbourg en 1868, en dehors du tracé de l'enceinte gallo-romaine.

Ces exemples montrent, je l'espère, par leur diversité la richesse de ce problème des rapports entre le château et la ville. Il reste à souhaiter que de telles études se multiplient avant de susciter une synthèse nécessaire.

André CHEDEVILLE

BERnos, Marcel, Femmes et gens d'Église dans la France classique, XVII -XVIII siècles, Préface de Jean Delumeau, Paris, Cerf, 2003, 404 p.

L'ouvrage de Marcel Bernos fondé sur des sources abondantes et une bonne bibliographie est un bel exemple d'érudition maîtrisée. C'est un travail d'historien de métier qui manifeste, comme ses écrits antérieurs, des qualités de réflexion prudente et de contestation judicieuse d'un certain nombre d'idées reçues. Il évite le piège de l'anachronisme, particulièrement redoutable pour le sujet qu'il a choisi. On ne saurait envisager l'histoire des femmes en chaussant les lunettes, roses ou noires selon les cas, des féministes contemporain(e)s. De même ce n'est évidemment pas en fonction de Vatican II que doit être étudiée l'Église tridentine, pas plus que la formation des Empires coloniaux ne doit l'être dans la perspective de la décolonisation... Marcel Bernos exorcise parfaitement ce danger majeur.

Le plan tripartite adopté (Le genre féminin - La femme dans tous ses " états " - Les gens d'église face aux femmes) est judicieux. Dans l'avant-propos sont dénoncées, avec raison, les schématisations abusives - ainsi le parallèle entre Bossuet et Fénelon, tendant à faire de ce dernier un féministe convaincu alors qu'en réalité $M$. de Meaux est plus libéral à l'égard de ses dirigées.

Cet ouvrage a été longuement mûri. En annexe (p. 353-354), l'auteur récapitule les publications qui en ont jalonné l'élaboration. Par un autre cheminement, M. Bernos retrouve souvent, dans le domaine de l'éducation féminine, les conclusions de la thèse de Linda Timmermans sur l'accès des femmes à la culture au $\mathrm{xvII}^{\mathrm{e}}$ siècle. Il rend un hommage mérité à cette chercheuse prématurément et dramatiquement disparue.

Dans la première partie, M. Bernos donne une lecture plus optimiste de la " pastorale de la peur ", émettant l'hypothèse plausible d'exagérations tonitruantes destinées à frapper fort l'esprit des pécheurs à convertir quitte à rassurer ultérieurement les scrupuleux. Un excellent chapitre est consacré à la " modestie ", vertu aussi masculine que féminine pour les clercs du XVII siècle mais réservée aux filles par les moralistes laics du temps des Lumières. La sorcellerie en France est, à juste titre, située par rapport aux autres pays d'Europe 
et à la Nouvelle Angleterre. Il me semble qu'il serait utile, après Michel de Certeau, d'insister sur la possession diabolique dans la perspective des rapports prêtre-femme dans le commerce triangulaire sorcier/femme/exorciste. Cette forme névropathique de la promotion de la femme par la religion est, certes, évoquée au chapitre Ix (les religieuses et " leurs hommes ") mais mériterait une évocation un peu plus longue que le développement de la page 227.

Jeunes filles, épouses et mères chrétiennes, veuves et religieuses sont l'objet de la seconde partie. Concernant les vocations religieuses, M. Bernos revient avec raison, en s'appuyant sur les travaux de Marie-A. Le Bourgeois, sur Anne de Xaintonge et son départ à Dôle en terre du roi d'Espagne, démarche inouïe pour une jeune femme, fille d'un conseiller à la cour du Parlement de Bourgogne. La vocation religieuse (féminine ou masculine) est souvent une rupture assumée avec le milieu familial. L'auteur rejoint ici les conclusions de Jean Delumeau à propos des visitandines (p. 117 et 317 et suiv.). Sur les femmes mariées, l'étude des manuels de confession, donne (p. 135), des résultats opposés aux conclusions de la thèse du Père Dhôtel sur les catéchismes (accroissement fort des développements sur le $6^{\mathrm{e}}$ commandement au XVII ${ }^{\mathrm{e}}$ siècle). M. Bernos note le fait, s'interdisant toute conclusion hâtive sur un dossier encore incomplètement exploré. En revanche pour les mères de famille, $M$. Bernos qui a judicieusement cité (p. 27 et 157-159) - à titre d'exemple de ce qu'il ne faut pas faire - le déplorable Amour en plus d'E. Badinter, émet des hypothèses qui sont totalement confirmées par la recherche micro-historique.

De bonnes pages sont consacrées aux veuves et au rôle important qu'elles ont joué dans la création religieuse du XVII ${ }^{\mathrm{e}}$ siècle. L'Ouest français est riche en spirituelles éminentes et en " couples mystiques" (ainsi Marie des Vallées et saint Jean Eudes, la carmélite tourangelle Madeleine de saint Joseph et Bérulle, le Père Joseph et Antoinette d'Orléans-Longueville créateurs des bénédictines du Calvaire). Marcel Bernos dont l'étude s'étend sur deux siècles ne pouvait évidemment pas traiter dans le détail toute cette matière humaine. Dans une belle galerie de portraits on retrouve la Tourangelle Marie de l'Incarnation introductrice des ursulines au Canada (le fils abandonné, le futur bénédictin Claude Martin n'avait alors que onze ans). Des colloques récents à Tours et au Québec viennent d'apporter de nouveaux éclairages sur la relation mère-fils (en particulier les communications de Jacques Maître ou de Joseph Baude). À propos d'une autre veuve, Madeleine de la Peltrie (1603-1671), également liée à la " croisade mystique " canadienne, les universités du Mans et de Caen ont organisé, en mai 2003 à Alençon son pays natal, des journées franco-canadiennes destinées à faire le point sur cette personnalité exceptionnelle qui - avec l'appui de la compagnie du Saint Sacrement - non contente de financer l'établissement des ursulines, partit avec elles en Nouvelle France où, tout en restant dans le siècle, elle continua à soutenir cette aventure religieuse. Cette veuve " simple laïque " comme disaient avec mépris les adversaires de la compagnie du Saint Sacrement, n'a pas connu la gloire historiographique de Marie de l'Incarnation. Il est vrai que, veuve sans enfant, elle n'avait pas comme l'ursuline un fils qui la contraignît à expliciter ses expériences intérieures.

Dans la troisième partie fort intéressante (par exemple à propos du théologien Bergier), des réflexions excellentes sur des " Lumières qui ne brillent pas pour les femmes " à verser au dossier des illusions du progrès! De même sur les confesseurs. Pour ceux qui " sollicitent " leurs pénitentes, il est impossible d'avoir des statistiques sûres - aussi la précision "normalement réprimés par les officialités " (p. 296) me paraît-elle un peu optimiste. L'affaire Benoîte Rencurel dont le dossier est passionnant vient d'attirer l'attention des historiens (en même 
temps que le présent ouvrage est paru un article consacré à cette mystique par Marie-Hélène Froeschlé-Chopard). Je souscris pleinement à la prudence de M. Bernos (p. 327) : "L'historien n'a pas à disserter sur la nature ou l'authenticité des visions. " (Dialogues supposés avec la Vierge ou objectivation d'un dialogue intérieur?). D'autant plus que les témoignages sont la traduction et la mise en forme par des juges ou des clercs d'un discours dont nous n'avons pas l'original.

Écrit de façon vivante, éclairé par de nombreuses notes infra-paginales, l'ouvrage de M. Bernos est un apport important à l'histoire des femmes dans l'Occident moderne. On regrettera seulement l'absence d'un index.

Malgré l'incontestable existence d'un antiféminisme d'une partie des clercs, malgré des reculs (le temps du " crépuscule des mystiques " dans la seconde moitié du XvII ${ }^{\mathrm{e}}$ siècle correspond à une diminution de l'influence des femmes dans l'Église), le Christianisme portait les germes d'une libération des filles d'Ève. Le riche dossier analysé par Marcel Bernos aide à comprendre pourquoi, comme le rappelle son préfacier Jean Delumeau " c'est dans les pays de tradition chrétienne que la femme aujourd'hui a le statut le plus enviable".

Robert SAUZET

GRANGER, Sylvie, Musiciens dans la ville (1650-1850), Paris, Belin, 2002, 320 p.

En introduction, l'auteur souligne la difficulté de cerner une activité par essence fugitive, aux sources dispersées, et un métier aux acteurs souvent gyrovagues, où la limite entre l'amateur et le professionnel reste longtemps floue. Dans le cadre du Maine, la première partie - "le métier de musicien " - traite des lieux et modes d'exécution musicale. Le titre nécessairement "fourre-tout " du premier chapitre - "scènes, tréteaux et parquets " - illustre d'emblée la difficulté de saisir dans une ville moyenne d'Ancien Régime des formes d'activités proLanes très inégalement enracinées, voire fragiles. Les tentatives pour établir au Mans un "Concert " public se soldent rapidement par autant d'échecs. Les représentations de théâtre musical par des troupes de passage sont très rares jusqu'à l'ouverture en 1776 de la salle de spectacle, financée par des actionnaires privés, qui accueille annuellement une dizaine d'opéras, d'opéras comiques ou de comédies mêlées d'ariettes. L'activité musicale est au contraire dynamisée au XIX ${ }^{\mathrm{e}}$ siècle par de nombreuses troupes et artistes de passage, phénomène européen dont les retombées locales sont aussi liées à l'essor du Mans (18000 habitants en 1801, 27000 en 1851), qui contraste avec la période précédente. Cette évolution accélère l'acculturation au goût musical parisien, par adjonction, non sans froissements parfois, des forces locales ou des amateurs aux musiciens professionnels ou extérieurs. Au bal privé s'ajoute à la fin de l'Ancien Régime le bal par abonnement; la saison s'allonge; les formes se diversifıent encore au XIX ${ }^{\mathrm{e}}$ siècle avec l'apparition du bal public, encore rare jusque-là.

Sous l'Ancien Régime, où le caractère discontinu, éparpillé de la vie musicale proLane ne permet pas d'en vivre, l'Église est le plus sûr employeur. Les chapitres de la cathédrale et de la collégiale Saint-Julien emploient une vingtaine de musiciens, chantres, maîtres de chapelle et organistes, auxquels s'ajoutent lors des grandes cérémonies des supplétifs. Douze églises paroissiales possèdent des orgues sous l'Ancien Régime, dont, il est vrai, les titulaires cumulent les postes. La Révolution oblige bien entendu ces musiciens, dont Sylvie Granger cerne au plus près les rémunérations en les resituant dans la gamme des exemples connus, 